\title{
Exact simulation of max-stable processes
}

\author{
Clément Dombry*, Sebastian Engelke ${ }^{\dagger}$ and Marco Oesting ${ }^{\ddagger}$
}

\begin{abstract}
Max-stable processes play an important role as models for spatial extreme events. Their complex structure as the pointwise maximum over an infinite number of random functions makes simulation highly nontrivial. Algorithms based on finite approximations that are used in practice are often not exact and computationally inefficient. We will present two algorithms for exact simulation of a max-stable process at a finite number of locations. The first algorithm generalizes the approach by Dieker and Mikosch [2015] for Brown-Resnick processes and it is based on simulation from the spectral measure. The second algorithm relies on the idea to simulate only the extremal functions, that is, those functions in the construction of a max-stable process that effectively contribute to the pointwise maximum. We study the complexity of both algorithms and prove that the second procedure is always more efficient. Moreover, we provide closed expressions for their implementation that cover the most popular models for max-stable processes and extreme value copulas. For simulation on dense grids, an adaptive design of the second algorithm is proposed.
\end{abstract}

Keywords: exact simulation; extremal function; extreme value distribution; max-stable process; spectral measure.

\section{Introduction}

Max-stable processes have become widely used tools to model spatial extreme events. Occurring naturally in the context of extremes as limits of maxima of independent copies of stochastic processes, they have found many applications in environmental sciences; see for instance Coles [1993], Buishand et al. [2008], Blanchet and Davison [2011], Davison et al. [2012].

Any sample continuous max-stable process $Z$ with unit Fréchet margins on some compact domain $\mathcal{X} \subset \mathbb{R}^{d}$ is characterized by a point process representation [de Haan, 1984]

$$
Z(x)=\max _{i \geq 1} \zeta_{i} \psi_{i}(x), \quad x \in \mathcal{X}
$$

${ }^{*}$ Université de Franche-Comté, Laboratoire de Mathématiques de Besançon, UMR CNRS 6623, 16 Route de Gray, 25030 Besançon cedex, France. Email: clement.dombry@univ-fcomte.fr

†École Polytechnique Fédérale de Lausanne, EPFL-FSB-MATHAA-STAT, Station 8, 1015 Lausanne, Switzerland. Faculté des Hautes Etudes Commerciales, Université de Lausanne, Extranef, UNIL-Dorigny, 1015 Lausanne, Switzerland. Email: sebastian.engelke@epfl.ch

${ }^{\ddagger}$ University of Twente, Faculty of Geo-Information Science and Earth Observation, PO Box 217, 7500 AE Enschede, The Netherlands. Email: m.oesting@utwente.nl 


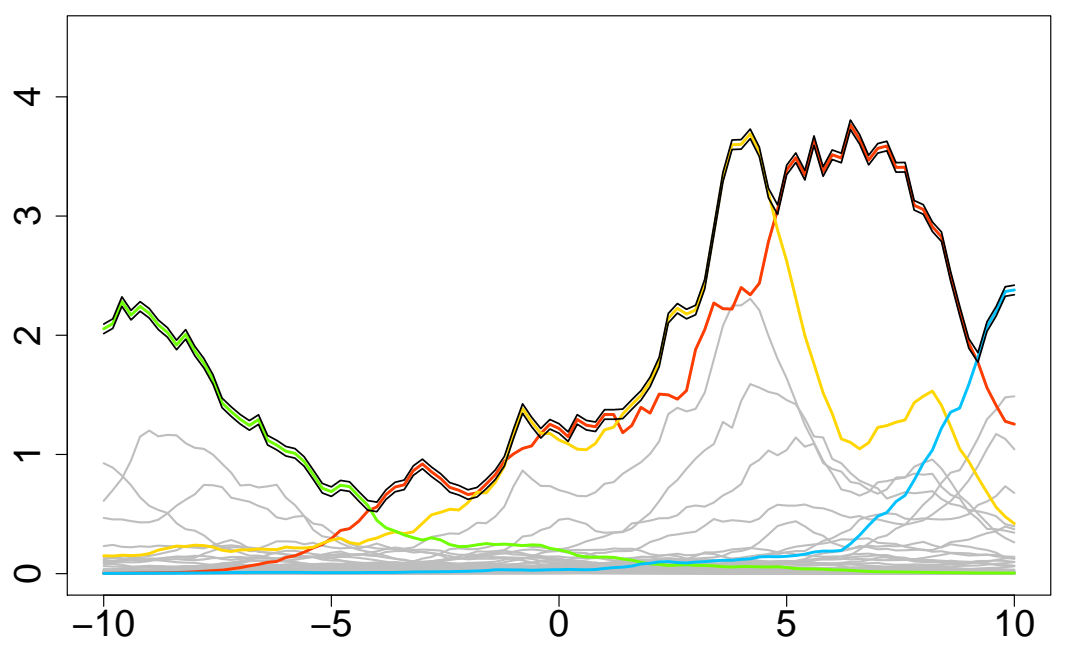

Figure 1: The Poisson point process $\left\{\left(\zeta_{i}, \psi_{i}\right), i \geq 1\right\}$ (grey). Only finitely many (colored) $\left(\zeta_{i}, \psi_{i}\right)$ contribute the maximum process $Z$ (bordered in black).

where $\left\{\left(\zeta_{i}, \psi_{i}\right), i \geq 1\right\}$ is a Poisson point process on $(0, \infty) \times \mathcal{C}$ with intensity measure $\zeta^{-2} \mathrm{~d} \zeta \times$ $\nu(\mathrm{d} \psi)$ for some locally finite measure $\nu$ on the space $\mathcal{C}=\mathcal{C}(\mathcal{X},[0, \infty))$ of continuous nonnegative functions on $\mathcal{X}$ such that

$$
\int_{\mathcal{C}} \psi(x) \nu(\mathrm{d} \psi)=1, \quad x \in \mathcal{X}
$$

Figure 1 shows a realization of $Z$ as a mixture of different random functions of the above point process. Due to this complex structure of max-stable processes, in many cases, analytical expressions are only available for lower-dimensional distributions and related characteristics need to be assessed by simulations. Moreover, non-conditional simulation is an important part of conditional simulation procedures that can be used to predict extreme events given some additional information [see Dombry et al., 2013, Oesting and Schlather, 2014, for example]. Thus, there is a need for fast and accurate simulation algorithms.

As the spectral representation (1) involves an infinite number of functions, exact simulation of $Z$ is in general not straightforward and finite approximations are used in practice. For the widely used Brown-Resnick processes [Kabluchko et al., 2009], for instance, Engelke et al. [2011] and Oesting et al. [2012] exploit the fact that the representation (1) is not unique in order to propose simulation procedures based on equivalent representations. However, often these approximations do not provide satisfactory results in terms of accuracy or computational effort. Exact simulation procedures can so far be implemented only in special cases. Schlather [2002] proposes an algorithm that simulates the points $\left\{\zeta_{i}, i \geq 1\right\}$ in (1) subsequently in a descending order until some stopping rule takes effect. If $\nu$ is the probability measure of a stochastic process whose supremum on $\mathcal{X}$ is almost surely bounded or if $Z$ is mixed moving maxima process with uniformly bounded and compactly supported shape function, this procedure allows for exact simulation of $Z$. For extremal- $t$ processes [Opitz, 2013], the elliptical structure of Gaussian processes can be exploited to obtain exact samples [Thibaud and Opitz, 2014]. Oesting et al. [2013] focus on a class of equivalent representations for general max-stable processes that, in principle, allow for exact simulation in an optimal way in terms of efficiency. They propose to simulate 
max-stable processes via the normalized spectral representation with all the spectral functions sharing the same supremum. Being efficient with respect to the number of spectral functions, the simulation of a single normalized spectral function might be rather intricate in some cases including the class of Brown-Resnick processes. For the latter, Dieker and Mikosch [2015] recently proposed a representation that enables exact simulation at finitely many locations.

Besides, several articles focus on the simulation of finite dimensional max-stable distributions or, equivalently, of their associated extreme value copula. Ghoudi et al. [1998] and Capéraà et al. [2000] propose simulation procedures for certain bivariate extreme value distributions. Stephenson [2003] considers the case of extreme value distributions of logistic type. Boldi [2009] provides a method for exact simulation from the spectral measure of extremal Dirichlet and logistic distributions.

In this paper, we propose two methods to simulate a general max-stable process $Z$ exactly at a finite number of locations. At first, we propose a generalization of the algorithm by Dieker and Mikosch [2015] showing that their approach relies on sampling from the spectral measure on the $L_{1}$-sphere of a multivariate extreme value distribution. The main idea of the second procedure is to simulate out of the infinite set $\left\{\zeta_{i} \psi_{i}, i \geq 1\right\}$ only the extremal functions [cf. Dombry and Éyi-Minko, 2012, 2013], i.e. those functions that satisfy $\zeta_{i} \psi_{i}(x)=Z(x)$ for some $x \in \mathcal{X}$ (the colored functions in Fig. 1). In contrast to all existing simulation procedures, the process $Z$ is not simulated simultaneously, but subsequently at different locations, rejecting all those functions that are not compatible with the process at the locations simulated so far. Both new procedures are based on random functions following the same type of distribution that can be easily simulated for most of the popular max-stable models. Our algorithms also apply very efficiently to exact simulation of finite-dimensional max-stable distributions or, equivalently, of the associated extreme value copulas.

\section{Extremal functions}

Without loss of generality, we may henceforth assume that $Z$ is a sample-continuous process with unit Fréchet margins given by the spectral representation (1). Indeed any samplecontinuous max-stable process can be obtained from a process with unit Fréchet margins via marginal transformations. We provide in this section some preliminaries on extremal functions and their distributions that will be essential in the new simulation methods. We use a point process approach and recall first that the $\mathcal{C}$-valued point process $\Phi=\left\{\phi_{i}\right\}_{i \geq 1}$ with $\phi_{i}=\zeta_{i} \psi_{i}$ is a Poisson point process with intensity

$$
\mu(A)=\int_{\mathcal{C}} \int_{0}^{\infty} 1_{\{\zeta \psi \in A\}} \zeta^{-2} \mathrm{~d} \zeta \nu(\mathrm{d} \psi), \quad A \subset \mathcal{C} \text { Borel. }
$$

Definition 1. Let $K \subset \mathcal{X}$ be a nonempty compact subset. A function $\phi \in \Phi$ is called $K$-extremal if there is some $x \in K$ such that $\phi(x)=Z(x)$, otherwise it is called $K$-subextremal. We denote by $\Phi_{K}^{+}$the set of $K$-extremal functions and by $\Phi_{K}^{-}$the set of $K$-subextremal functions.

It can be shown that $\Phi_{K}^{+}$and $\Phi_{K}^{-}$are properly defined point process. When $K=\left\{x_{0}\right\}, x_{0} \in \mathcal{X}$, is reduced to a single point, it is easy to show that $\Phi_{\left\{x_{0}\right\}}^{+}$is also almost surely reduced to a single point that we denote by $\phi_{x_{0}}^{+}$, termed the extremal function at point $x_{0}$. The distribution of $\phi_{x_{0}}^{+}$is given in the next proposition [see Proposition 4.2 in Dombry and Éyi-Minko, 2013]. 
Proposition 1. The random variables $Z\left(x_{0}\right)$ and $\phi_{x_{0}}^{+} / Z\left(x_{0}\right)$ are independent. Furthermore, $Z\left(x_{0}\right)$ has a unit Fréchet distribution and the distribution of $\phi_{x_{0}}^{+} / Z\left(x_{0}\right)$ is

$$
P_{x_{0}}(A)=\operatorname{pr}\left(\phi_{x_{0}}^{+} / Z\left(x_{0}\right) \in A\right)=\int_{\mathcal{C}} 1_{\left\{f / f\left(x_{0}\right) \in A\right\}} f\left(x_{0}\right) \nu(\mathrm{d} f), \quad A \subset \mathcal{C} \text { Borel } .
$$

By definition, $\phi_{x_{0}}^{+}\left(x_{0}\right)=Z\left(x_{0}\right)$. This entails that the distribution $P_{x_{0}}$ is supported by the subset of functions $\left\{f \in \mathcal{C}, f\left(x_{0}\right)=1\right\}$.

Proposition 2. The restricted point process

$$
\Phi \cap\left\{f \in \mathcal{C}, f\left(x_{0}\right)>0\right\}
$$

is a Poisson point process with intensity

$$
\int_{A} 1_{\left\{f\left(x_{0}\right)>0\right\}} \mu(\mathrm{d} f)=\int_{\mathcal{C}} \int_{0}^{\infty} 1_{\{\zeta f \in A\}} \zeta^{-2} \mathrm{~d} \zeta P_{x_{0}}(\mathrm{~d} f), \quad A \subset \mathcal{C} \text { Borel } .
$$

Proof. The fact that the restricted point process $\Phi \cap\left\{f \in \mathcal{C}, f\left(x_{0}\right)>0\right\}$ is a Poisson point process with intensity $1_{\left\{f\left(x_{0}\right)>0\right\}} \mu(\mathrm{d} f)$ is standard. We prove Equation (5). For $A \subset \mathcal{C}$ Borel,

$$
\begin{aligned}
\int_{\mathcal{C}} \int_{0}^{\infty} 1_{\{\zeta f \in A\}} \zeta^{-2} \mathrm{~d} \zeta P_{x_{0}}(\mathrm{~d} f)=\int_{\mathcal{C}} \int_{0}^{\infty} 1_{\left\{\zeta f / f\left(x_{0}\right) \in A\right\}} \zeta^{-2} \mathrm{~d} \zeta f\left(x_{0}\right) \nu(\mathrm{d} f) \\
=\int_{\mathcal{C}} \int_{0}^{\infty} 1_{\{\tilde{\zeta} f \in A\}} \tilde{\zeta}^{-2} \mathrm{~d} \tilde{\zeta} 1_{\left\{f\left(x_{0}\right)>0\right\}} \nu(\mathrm{d} f)=\int_{\mathcal{C}} 1_{\{f \in A\}} 1_{\left\{f\left(x_{0}\right)>0\right\}} \mu(\mathrm{d} f)
\end{aligned}
$$

Here, we use successively Eq. (4), the change of variable $\tilde{\zeta}=\zeta / f\left(x_{0}\right)$ with $f\left(x_{0}\right)>0$ and Eq. (3) for the last equality.

REMARK 1. As a consequence of (5), independent copies $Y_{i}, i \geq 1$, of processes with distribution $P_{x_{0}}$ result in a point process $\left\{\zeta_{i} Y_{i}\right\}_{i \geq 1}$ which has the same distribution as the restricted point process $\Phi \cap\left\{f \in \mathcal{C}, f\left(x_{0}\right)>0\right\}$. If $\nu\left(\left\{f \in \mathcal{C}, f\left(x_{0}\right)=0\right\}\right)=0$, then $\Phi$ consists only of functions with positive value at $x_{0}$ and $\Phi$ has the same distribution as $\left\{\zeta_{i} Y_{i}\right\}_{i \geq 1}$. This provides an alternative point process representation of the max-stable process $Z$ in terms of a random process $Y$ such that $Y\left(x_{0}\right)=1$ almost surely. In Engelke et al. [2014] and Engelke et al. [2015], this representation is exploited for statistical inference of $Z$.

It follows clearly from Definition 1 that we have the decomposition $\Phi=\Phi_{K}^{+} \cup \Phi_{K}^{-}$. The following proposition will play a crucial role in our second approach based on extremal functions. If $f_{1}, f_{2}$ are two functions on $\mathcal{X}$, the notation $f_{1}<_{K} f_{2}$ means $f_{1}(x)<f_{2}(x)$ for all $x \in K$.

Proposition 3 (Dombry and Éyi-Minko [2012], Lemma 3.2). The conditional distribution of $\Phi_{K}^{-}$with respect to $\Phi_{K}^{+}$is equal to the distribution of a Poisson point process on $\mathcal{C}$ with intensity $1_{\left\{f<{ }_{K} Z\right\}} \mu(d f)$. 


\section{Exact simulation procedures}

\subsection{Introduction}

Recently, Dieker and Mikosch [2015] provided a new representation of stationary BrownResnick processes that allows for exact simulation of their finite-dimensional distributions. In this section we introduce two methods for exact simulation of arbitrary max-stable processes and distributions. More precisely, for a fixed number $N \in \mathbb{N}$ of pairwise distinct locations $x=\left(x_{1}, \ldots, x_{N}\right) \in \mathcal{X}^{N}$, we aim at obtaining exact simulation of the max-stable random vector

$$
Z(x)=\left(Z\left(x_{1}\right), \ldots, Z\left(x_{N}\right)\right) .
$$

Both procedures are intimately connected with the distribution $P_{x}$ in (4).

The first method extends the Dieker and Mikosch [2015] approach. In fact, we show that their representation is nothing else than the so-called spectral representation of the Brown-Resnick process, and their procedure actually enables exact simulation from the spectral measure on the $L_{1}$-sphere. In Section 3.2 we derive a way of simulating from the spectral measure of a general max-stable distribution or a possibly non-stationary max-stable process.

The second procedure presented in Section 3.3 relies on conditional distributions of the Poisson point process underlying any max-stable process. This approach also allows for exact simulation of (6) by simulating at each location only the unique function that actually attains the maximum; see Fig. 1. It is intuitive and turns out to be even more powerful than the spectral method.

\subsection{Simulation via the spectral measure}

Let us recall the spectral decomposition of the max-stable random vector $Z(x)$; for details we refer to Resnick [2008, Chap. 5]. Here, we write $f(x)=\left(f\left(x_{1}\right), \ldots, f\left(x_{N}\right)\right)$ for the restriction of a generic (random) function $f$ to the locations $x \in \mathcal{X}^{N}$. Following Equation (1), the maxstable random vector $Z(x)$ is generated by the Poisson point process $\Phi_{x}=\left\{\zeta_{i} \psi_{i}(x), i \geq 1\right\}$ whose intensity measure on the cone $E=[0, \infty)^{N}$ is denoted by $\mu_{x}$. Due to its homogeneity, the exponent measure $\mu_{x}$ can be factorized into a radial part on $(0, \infty)$ and an angular part on the unit $L_{1}$-sphere $S_{N-1}=\{z \in E:\|z\|=1\}$, where $\|z\|=z_{1}+\cdots+z_{N}$, for $z=\left(z_{1}, \ldots, z_{N}\right) \in$ $E$. More precisely, a change to polar coordinates under the map $U: E \rightarrow(0, \infty) \times S_{N-1}$, $U(z)=(\|z\|, z /\|z\|)$ yields

$$
\mu_{x}(F)=\int_{U(F)} \mu_{x} \circ U^{-1}(\mathrm{~d} r, \mathrm{~d} s)=N \int_{U(F)} r^{-2} \mathrm{~d} r H(\mathrm{~d} s)
$$

for any Borel subset $F \subset E$. The probability measure $H$ on $S_{N-1}$ is called spectral measure of $Z(x)$ and it satisfies

$$
\int_{S_{N-1}} s_{j} H(d s)=N^{-1}, \quad j=1, \ldots, N
$$

Equation (7) shows that we can represent the process $\Phi_{x}$ as

$$
\Phi_{x}=\left\{U^{-1}\left(R_{i}, Q_{i}\right): i \geq 1\right\}=\left\{R_{i} Q_{i}: i \geq 1\right\}
$$


where $\left\{R_{i}: i \geq 1\right\}$ is a Poisson point process on $(0, \infty)$ with intensity $N r^{-2} \mathrm{~d} r$ and $Q_{i}$, $i \geq 1$, are independent samples from the spectral measure $H$ on $S_{N-1}$. The great advantage of this representation is that the components of $Q_{i}$ are bounded by 1 . This ensures that $Z(x)=$ $\max _{i \geq 1} R_{i} Q_{i}$ can be simulated exactly by generating the largest $R_{i}$ first until no more of the remaining points $R_{i} Q_{i}$ can contribute to the maximum.

The only difficulty is thus to generate the random variables $Q_{i}$ from the probability measure $H$ on the $(N-1)$-dimensional positive sphere $S_{N-1}$. The following theorem gives the solution to this problem for the max-stable distribution $Z(x)$.

Theorem 1. Let $T_{i}, i \geq 1$, be independent copies of a random variable $T$ with uniform distribution on the discrete set $\{1, \ldots, N\}$. Further, for any $k=1, \ldots, N$, let $Y_{i}^{(k)}, i \geq 1$, be independent random processes with distribution $P_{x_{k}}$ as in (4). Then, the $S_{N-1}$-valued random variables

$$
Q_{i}=\frac{Y_{i}^{\left(T_{i}\right)}(x)}{\left\|Y_{i}^{\left(T_{i}\right)}(x)\right\|}, \quad i \geq 1,
$$

are independent with distribution $H$. Consequently, with $\left\{R_{i}, i \geq 1\right\}$ as above,

$$
Z(x)=\max _{i \geq 1} R_{i} \frac{Y_{i}^{\left(T_{i}\right)}(x)}{\left\|Y_{i}^{\left(T_{i}\right)}(x)\right\|} .
$$

Proof. For any $k=1, \ldots, N$, Eq. (4) implies

$$
\int_{\mathcal{C}} f\left(x_{k}\right) 1_{\{f(x) /\|f(x)\| \in A\}} \nu(\mathrm{d} f)=\int_{\mathcal{C}} 1_{\{f(x) /\|f(x)\| \in A\}} P_{x_{k}}(\mathrm{~d} f) .
$$

We compute the $\mu_{x}$-measure of the set $U^{-1}((u, \infty) \times A)$ for $u>0$ and a Borel set $A \subset S_{N-1}$.

$$
\begin{aligned}
\mu_{x}\left(U^{-1}((u, \infty) \times A)\right)=\int_{\mathcal{C}} \int_{0}^{\infty} 1_{\{\zeta\|f(x)\|>u\}} 1_{\{f(x) /\|f(x)\| \in A\}} \zeta^{-2} \mathrm{~d} \zeta \nu(\mathrm{d} f) \\
=\frac{1}{u} \int_{\mathcal{C}}\|f(x)\| 1_{\{f(x) /\|f(x)\| \in A\}} \nu(\mathrm{d} f)=\frac{1}{u} \sum_{k=1}^{N} \int_{\mathcal{C}} f\left(x_{k}\right) 1_{\{f(x) /\|f(x)\| \in A\}} \nu(\mathrm{d} f) \\
=\frac{1}{u} \sum_{k=1}^{N} \int_{\mathcal{C}} 1_{\{f(x) /\|f(x)\| \in A\}} P_{x_{k}}(\mathrm{~d} f)=\frac{N}{u} \cdot \frac{1}{N} \sum_{k=1}^{N} \int_{\mathcal{C}} 1_{\{f(x) /\|f(x)\| \in A\}} P_{x_{k}}(\mathrm{~d} f),
\end{aligned}
$$

where the second last equation follows from $(8)$. Let $Y^{(k)}, k=1, \ldots, N$, be independent random processes with distribution $P_{x_{k}}$, respectively, and let $T$ be an independent uniform random variable on $\{1, \ldots, N\}$, then the above implies that

$$
\mu_{x}\left(U^{-1}((u, \infty) \times A)\right)=\frac{N}{u} \cdot \operatorname{pr}\left\{\frac{Y^{(T)}(x)}{\left\|Y^{(T)}(x)\right\|} \in A\right\} .
$$

Comparing this with (7) yields the assertion of the theorem.

Theorem 1 shows how to simulate from the spectral measure $H$. It requires only to be able to simulate from the distributions $P_{x_{k}}, k=1, \ldots, N$. Algorithm 1 , an adaptation of Schlather's [2002] algorithm, provides an exact sample from the max-stable process $Z$ at locations $x$.

REMARK 2. The results on the distribution $P_{x_{k}}$ for stationary Brown-Resnick processes obtained in Subsection 5.2 reveal that Algorithm 1 is identical to the algorithm by Dieker and Mikosch 2015] in this case. 


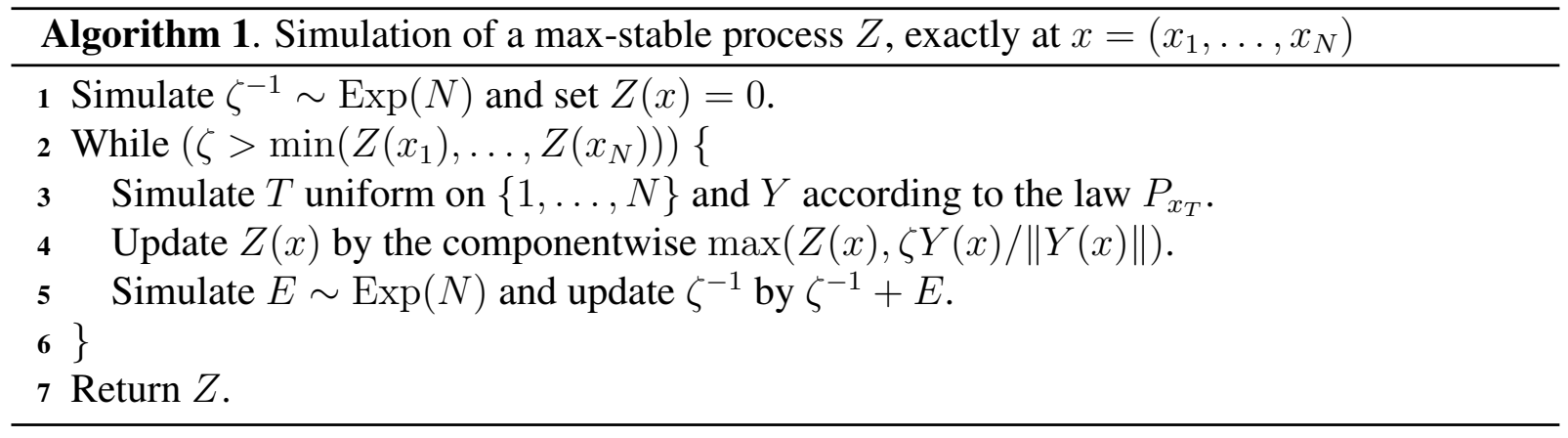

\subsection{Simulation via extremal functions}

We now introduce the second procedure for exact simulation of the max-stable process $Z$ at locations $x=\left(x_{1}, \ldots, x_{N}\right) \in \mathcal{X}^{N}$. For $n=1, \ldots, N$ we consider the extremal and subextremal point processes $\Phi_{n}^{+}=\Phi_{\left\{x_{1}, \ldots, x_{n}\right\}}^{+}$and $\Phi_{n}^{-}=\Phi_{\left\{x_{1}, \ldots, x_{n}\right\}}^{-}$. We have $\Phi_{n}^{+}=\left\{\phi_{x_{i}}^{+}\right\}_{1 \leq i \leq n}$ but there may be some repetitions in the right-hand side. We define the $n$ th-step maximum process

$$
Z_{n}(x)=\max _{\phi \in \Phi_{n}^{+}} \phi(x)=\max _{1 \leq i \leq n} \phi_{x_{i}}^{+}(x), \quad x \in \mathcal{X} .
$$

By the definition of extremal functions we have $Z\left(x_{i}\right)=\phi_{x_{i}}^{+}\left(x_{i}\right)$ and clearly

$$
Z\left(x_{i}\right)=Z_{n}\left(x_{i}\right), \quad i=1, \ldots, n
$$

Hence, in order to exactly simulate $Z$ at locations $x$, it is enough to exactly simulate $\Phi_{N}^{+}$. We will proceed inductively and simulate the sequence $\left(\phi_{x_{n}}^{+}\right)_{1 \leq n \leq N}$ according to the following theorem.

Theorem 2. The distribution of $\left(\phi_{x_{n}}^{+}\right)_{1 \leq n \leq N}$ is given as follows:

- Initial distribution: the extremal function $\phi_{x_{1}}^{+}$has the same distribution as $F_{1} Y_{1}$ where $F_{1}$ is a unit Fréchet random variable and $Y_{1}$ an independent random process with distribution $P_{x_{1}}$ given by (4).

- Conditional distribution: for $1 \leq n \leq N-1$, the conditional distribution of $\phi_{x_{n+1}}^{+}$with respect to $\left(\phi_{x_{i}}^{+}\right)_{1 \leq i \leq n}$ is equal to the distribution of

$$
\tilde{\phi}_{x_{n+1}}^{+}= \begin{cases}\operatorname{argmax}_{\phi \in \tilde{\Phi}_{n+1}} \phi\left(x_{n+1}\right) & \text { if } \tilde{\Phi}_{n+1} \neq \emptyset \\ \operatorname{argmax}_{\phi \in \Phi_{n}^{+}} \phi\left(x_{n+1}\right) & \text { if } \tilde{\Phi}_{n+1}=\emptyset\end{cases}
$$

where $\tilde{\Phi}_{n+1}$ is a Poisson point process with intensity

$$
1_{\left\{f\left(x_{i}\right)<Z_{n}\left(x_{i}\right), 1 \leq i \leq n\right\}} 1_{\left\{f\left(x_{n+1}\right)>Z_{n}\left(x_{n+1}\right)\right\}} \mu(\mathrm{d} f)
$$

and $Z_{n}$ is defined by (9).

Proof. The distribution of $\phi_{x_{1}}^{+}$is given in Proposition 1. We prove the result for the conditional distribution of $\phi_{x_{n+1}}^{+}$with respect to $\left(\phi_{x_{i}}^{+}\right)_{1 \leq i \leq n}$. Recall that $\Phi_{n}^{+}=\left\{\phi_{x_{1}}^{+}, \ldots, \phi_{x_{n}}^{+}\right\}$and that 
according to Proposition 3, the conditional distribution of $\Phi_{n}^{-}$with respect to $\Phi_{n}^{+}$is equal to the distribution of a Poisson point process with intensity

$$
1_{\left\{f\left(x_{i}\right)<Z\left(x_{i}\right), 1 \leq i \leq n\right\}} \mu(\mathrm{d} f)=1_{\left\{f\left(x_{i}\right)<Z_{n}\left(x_{i}\right), 1 \leq i \leq n\right\}} \mu(\mathrm{d} f),
$$

where the equality follows from Eq. (10). In order to determine $\phi_{x_{n+1}}^{+}$we focus on the functions $\phi \in \Phi_{n}^{-}$satisfying $\phi\left(x_{n+1}\right)>Z_{n}\left(x_{n+1}\right)$ and consider the restriction

$$
\tilde{\Phi}_{n+1}=\Phi_{n}^{-} \cap\left\{f \in \mathcal{C}, f\left(x_{n+1}\right)>Z_{n}\left(x_{n+1}\right)\right\} .
$$

It follows from Eq. (12) that conditionally on $\left(\phi_{x_{i}}^{+}\right)_{1 \leq i \leq n}, \tilde{\Phi}_{n+1}$ is a Poisson point process with intensity given by Eq. (11). We distinguish two cases:

- if $\tilde{\Phi}_{n+1}=\emptyset$ then there is no function in $\Phi_{n}^{-}$exceeding $Z_{n}$ at point $x_{n+1}$, that is, $Z\left(x_{n+1}\right)=$ $Z_{n}\left(x_{n+1}\right)$ and $\phi_{x_{n+1}}^{+}=\operatorname{argmax}_{\phi \in \Phi_{n}^{+}} \phi\left(x_{n+1}\right)$.

- If $\tilde{\Phi}_{n+1} \neq \emptyset$ then there is some function in $\Phi_{n}^{-}$exceeding $Z_{n}$ at point $x_{n+1}$, that is, $Z\left(x_{n+1}\right)>Z_{n}\left(x_{n+1}\right)$ and $\phi_{x_{n+1}}^{+}=\operatorname{argmax}_{\phi \in \tilde{\Phi}_{n+1}} \phi\left(x_{n+1}\right)$.

This concludes the proof of Theorem 2

From the above theorem, one can deduce Algorithm 2 for exact simulation of the max-stable process $Z$ at locations $x=\left(x_{1}, \ldots, x_{N}\right)$. According to Proposition 2 and Remark 1, the distribution $P_{x_{n+1}}$ can be used to simulate $\tilde{\Phi}_{n+1}$ with intensity (11). Hence, as for the spectral method, the second algorithm requires only to be able to simulate from the distributions $P_{x_{n}}$, $n=1, \ldots, N$. Figure 2 illustrates the procedure.

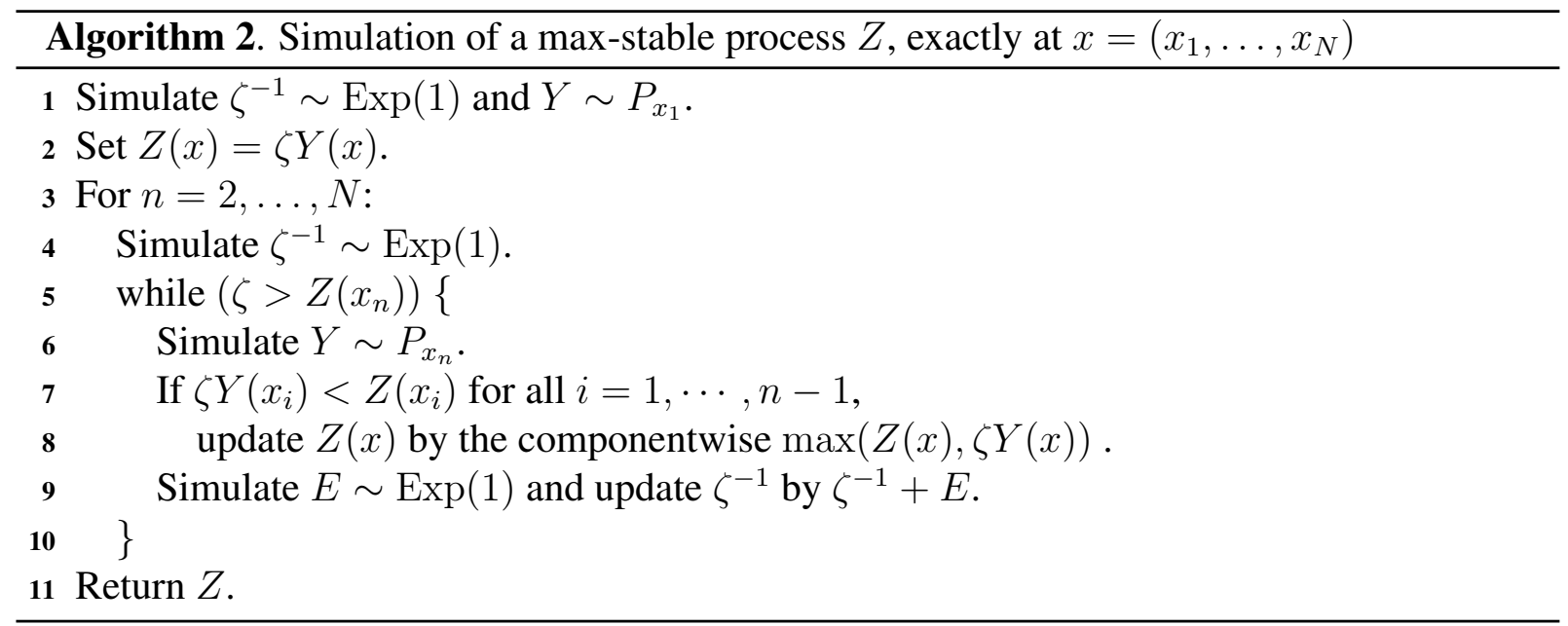

\section{Complexity of the Algorithms}

In this section, we aim at assessing the complexity of Algorithms 1 and 2 as a function of the number $N$ of simulation sites. Both algorithms contain the simulation of exponential random 

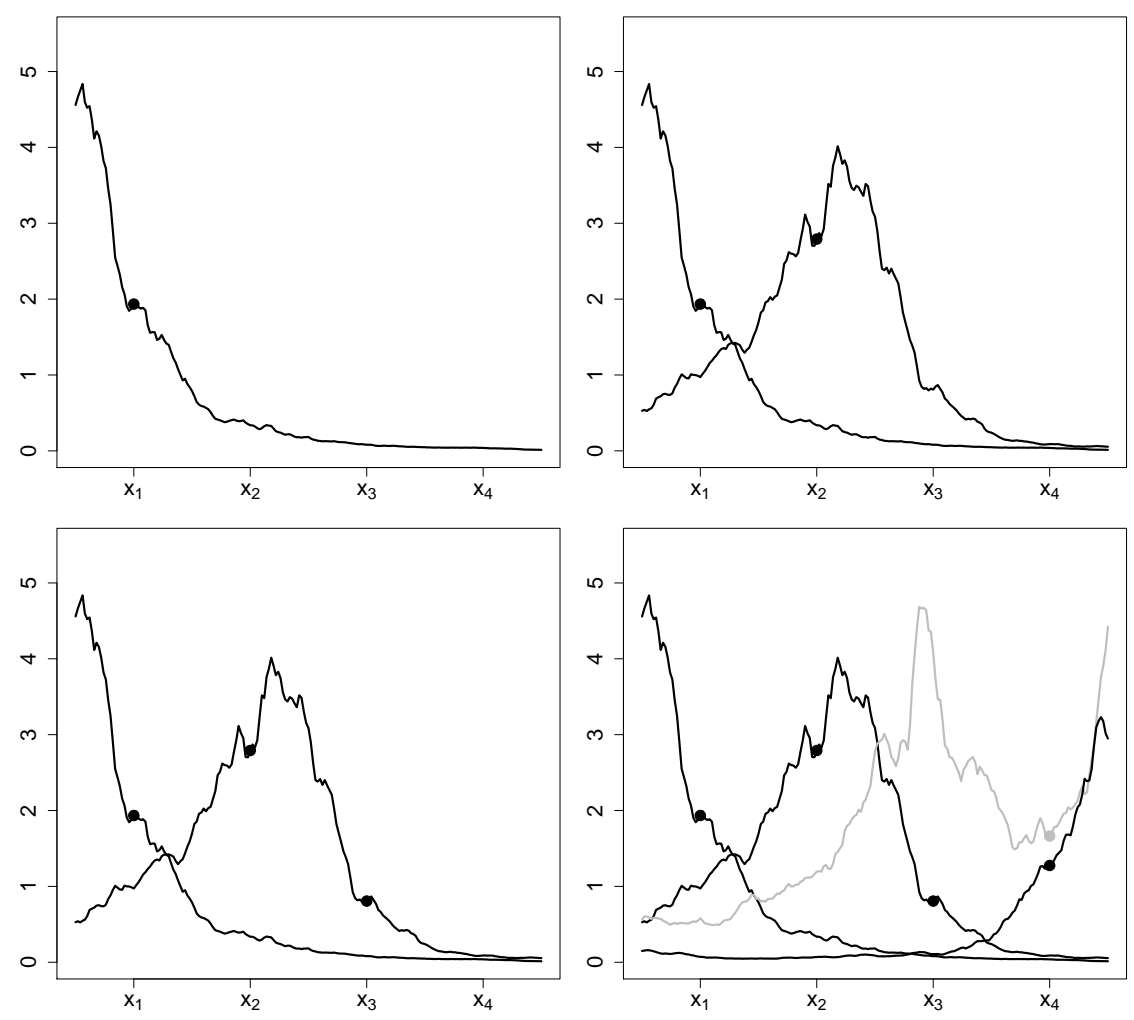

Figure 2: Simulation of $Z$ via Algorithm 2 at locations $\left(x_{1}, x_{2}, x_{3}, x_{4}\right)$. Initial process $\phi_{x_{1}}^{+}$is always accepted (top-left). Second process $\phi_{x_{2}}^{+}$is accepted as it exceeds $Z_{1}=\phi_{x_{1}}^{+}$at $x_{2}$ but not at $x_{1}$ (top-right). Third process $\phi_{x_{3}}^{+}$is equal to $\phi_{x_{2}}^{+}$since $\tilde{\Phi}_{3}=\emptyset$ (bottom-left). First sample of $P_{x_{4}}$ (grey line) is rejected since it exceeds $Z_{3}$ at $x_{3}$; second sample is valid and thus called $\phi_{x_{4}}^{+}$ (bottom-right).

variables $E$ and the simulation of $N$-dimensional random vectors $Y(x)$ according to a mixture of the laws $P_{x_{1}}, \ldots, P_{x_{N}}$. The simulation of $E$ causes much less computational effort than the simulation of $Y$ and can therefore be neglected in the analysis of the algorithmic complexity. We thus consider the number $C_{1}(N)$ and $C_{2}(N)$ of random vectors $Y(x)$ that need to be simulated by Algorithm 1 and 2 respectively to obtain one exact simulation of $Z(x)$. Interestingly, one can provide simple expressions for $\mathrm{E}\left(C_{1}(N)\right)$ and $\mathrm{E}\left(C_{2}(N)\right)$.

Proposition 4. The expected number of random vectors $Y(x)$ that are needed for exact simulation of $Z$ at $x=\left(x_{1}, \ldots, x_{N}\right)$ are:

Algorithm 1: $\quad \mathrm{E}\left(C_{1}(N)\right)=N \mathrm{E}\left(\max _{i=1}^{N} Z\left(x_{i}\right)^{-1}\right)$

Algorithm 2: $\quad \mathrm{E}\left(C_{2}(N)\right)=N$

Furthermore, $\mathrm{E}\left(C_{1}(N)\right) \geq \mathrm{E}\left(C_{2}(N)\right)$ with equality if and only if $Z\left(x_{1}\right)=\ldots=Z\left(x_{N}\right)$ almost surely.

The expectation of $C_{1}(N)$ can be calculated similarly to Proposition 4.8 in Oesting et al. [2013]. 
The proof for the expectation of $C_{2}(N)$ is more difficult and, for the sake of brevity, it is provided as a supplementary material to this paper.

We conclude this section with some comments on the complexity of our algorithms and a comparison with other exact simulation procedures. Proposition 4 shows that, for any max-stable process, Algorithm 2 is more efficient than Algorithm 1 in terms of the expected number of simulated functions. As the spectral functions follow either one of the laws $P_{x_{1}}, \ldots, P_{x_{N}}$ or a mixture of these, the simulation of a single spectral function is equally complex in both cases. Thus, the new Algorithm 2 is always preferable to the generalized Dieker-Mikosch algorithm, Algorithm 1

Next, we compare Algorithm 2 with the exact simulation algorithm via the normalized spectral representation proposed by Oesting et al. [2013]. By Proposition 4.8 in Oesting et al. [2013], the number $C_{3}(N)$ of simulated normalized spectral functions satisfies

$$
\mathrm{E}\left(C_{3}(N)\right)=\left(\int \max _{i=1}^{N} \psi\left(x_{i}\right) \nu(\mathrm{d} \psi)\right) \mathrm{E}\left(\max _{i=1}^{N} Z\left(x_{i}\right)^{-1}\right)
$$

and, thus, depends both on the geometry of the set $\left\{x_{1}, \ldots, x_{N}\right\}$ and on the law of the maxstable process $Z$. For simulation on a large and dense subset of $\mathcal{X}$, the algorithm via the normalized spectral representation is more efficient than Algorithm 2 as $\mathrm{E}\left(C_{3}(N)\right)$ is bounded by $\left(\int \sup _{x \in \mathcal{X}} \psi(x) \nu(\mathrm{d} \psi)\right) \mathrm{E}\left(\sup _{x \in \mathcal{X}} Z(x)^{-1}\right)$ while $\mathrm{E}_{2}(N)=N$ grows with the size of the subset. If $N$ is small or moderate and the vector $Z(x)$ is weakly dependent, we may also have $\mathrm{E}\left(C_{2}(N)\right)<\mathrm{E}\left(C_{3}(N)\right)$.

Besides the efficiency in terms of the expected number of simulated functions, we also need to take into account the complexity of the simulation of a single spectral function. Exact and efficient simulation procedures for the normalized spectral function are known for some cases only (such as moving maxima processes), while they are not available in other cases like BrownResnick or extremal- $t$ processes. In contrast, the random functions in Algorithms 1 and 2 with distributions $P_{x_{0}}$ in (4), $x_{0} \in \mathcal{X}$, can be simulated efficiently for the most popular max-stable process and extreme value copula models. Indeed, in Section 5 below we provide closed form expressions for various important examples.

\section{Examples}

\subsection{Moving maximum process}

The parameter space is $\mathcal{X}=\mathbb{Z}^{d}$ or $\mathbb{R}^{d}$ and $\lambda$ denotes the counting measure or the Lebesgue measure, respectively. A moving maximum process on $\mathcal{X}$ is a max-stable process of the form

$$
Z(x)=\max _{i \geq 1} \zeta_{i} h\left(x-\chi_{i}\right), \quad x \in \mathcal{X}
$$

where $\left\{\left(\zeta_{i}, \chi_{i}\right), i \geq 1\right\}$ is a Poisson point process on $(0, \infty) \times \mathcal{X}$ with intensity measure $\zeta^{-2} \mathrm{~d} \zeta \times$ $\lambda(\mathrm{d} \chi)$ and $h: \mathcal{X} \rightarrow[0, \infty)$ is a continuous function satisfying $\int_{\mathcal{X}} h(x) \lambda(\mathrm{d} x)=1$. A famous example is the Gaussian extreme value process where $h$ is a multivariate Gaussian density on $\mathbb{R}^{d}[$ Smith, 1990]. 
Proposition 5. Consider the moving maximum process (13). For all $x_{0} \in \mathcal{X}$, the distribution $P_{x_{0}}$ is equal to the distribution of the random function

$$
\frac{h\left(\cdot+\chi-x_{0}\right)}{h(\chi)} \text { with } \chi \sim h(u) \lambda(\mathrm{d} u) .
$$

All proofs of this section can be found in the supplementary material to this paper.

\subsection{Brown-Resnick process}

We consider max-stable processes obtained by representation (1) where $\nu$ is a probability measure on $\mathcal{C}$ given by

$$
\nu(A)=\operatorname{pr}\left[\exp \left(W(\cdot)-\sigma^{2}(\cdot) / 2\right) \in A\right], \quad A \subset \mathcal{C} \text { Borel }
$$

with $W$ a sample-continuous centered Gaussian process on $\mathcal{X}$ with variance $\sigma^{2}(x)=\mathrm{E}\left[W(x)^{2}\right]$. In other words, $\nu$ is the distribution of the log-normal process $Y(x)=\exp \left(W(x)-\sigma^{2}(x) / 2\right)$, $x \in \mathcal{X}$. The relation $\mathrm{E}[\exp \{W(x)\}]=\exp \left(\sigma^{2}(x) / 2\right)$ ensures that $\mathrm{E}[Y(x)]=1$ and Equation (2) is satisfied.

An interesting phenomenon arises when $\mathcal{X}=\mathbb{Z}^{d}$ or $\mathbb{R}^{d}$ and $W$ has stationary increments: Kabluchko et al. [2009] show that the associated max-stable process $Z$ is then stationary with distribution depending only on the semi-variogram

$$
\gamma(h)=\frac{1}{2} \mathrm{E}\left[\{W(h)-W(0)\}^{2}\right], \quad h \in \mathcal{X} .
$$

The stationary max-stable process $Z$ is called a Brown-Resnick process. However, our results apply both in the stationary and non-stationary case [cf., Kabluchko, 2011] and except stated otherwise we do not assume that $W$ has stationary increments.

Proposition 6. Consider the Brown-Resnick type model (14). For all $x_{0} \in \mathcal{X}$, the distribution $P_{x_{0}}$ is equal to the distribution of the log-normal process

$$
\tilde{Y}(x)=\exp \left(W(x)-W\left(x_{0}\right)-\frac{1}{2} \operatorname{Var}\left[W(x)-W\left(x_{0}\right)\right]\right), \quad x \in \mathcal{X} .
$$

REMARK 3. It is easy to deduce from the proposition that in the Brown-Resnick case where $W$ has stationary increments, then $\widetilde{Y}$ has the same distribution as

$$
\exp \left(W\left(x-x_{0}\right)-W(0)-\frac{1}{2} \gamma\left(x-x_{0}\right)\right), \quad x \in \mathcal{X}
$$

REMARK 4. The finite dimensional margins of Brown-Resnick processes are Hüsler-Reiss distributions [cf., Hüsler and Reiss, 1989] and the above therefore provides a method for their exact simulation. 


\subsection{Extremal- $t$ process}

We consider the so called extremal- $t$ max-stable process [cf., Opitz, 2013] defined by representation (1) with $\nu$ the distribution of the random process

$$
Y(x)=c_{\alpha} \max (0, W(x))^{\alpha}, \quad x \in \mathcal{X},
$$

where $\alpha>0, c_{\alpha}=\pi^{1 / 2} 2^{-(\alpha-2) / 2} / \Gamma\left(\frac{1+\alpha}{2}\right)$, and $W$ a sample-continuous centered Gaussian process on $\mathcal{X}$ with unit variance and covariance function $c$. The constant $c_{\alpha}$ is such that $\mathrm{E}[Y(x)] \equiv 1$ so that Equation (2) is satisfied. For $\alpha=1$, the corresponding max-stable process in (1) coincides with the widely used extremal Gaussian process by Schlather [2002].

Proposition 7. Consider the extremal-t model (15). For all $x_{0} \in \mathcal{X}$, the distribution $P_{x_{0}}$ is equal to the distribution of $\max (T, 0)^{\alpha}$, where $T=(T(x))_{x \in \mathcal{X}}$ is a Student process with $\alpha+1$ degrees of freedom, location and scale functions given respectively by

$$
\mu(x)=c\left(x_{0}, x\right) \quad \text { and } \quad \hat{c}\left(x_{1}, x_{2}\right)=\frac{c\left(x_{1}, x_{2}\right)-c\left(x_{0}, x_{1}\right) c\left(x_{0}, x_{2}\right)}{(\alpha+1)} .
$$

\subsection{Multivariate extreme value distributions}

In this section, we review some popular models for multivariate extreme value distributions, i.e., the case when $\mathcal{X}=\{1, \ldots, N\}$ in (1) is a finite set for some fixed $N \in \mathbb{N}$. For these models, we explicitly calculate the measure $P_{j_{0}}$ for any $j_{0}=1, \ldots, N$. Unless otherwise stated, all random vectors are $N$-dimensional in this section. Multivariate extreme value distributions differ from extreme value copulas only by a change in the marginal distribution, so that our methodology applies directly to exact simulation of extreme value copulas. For more details on the models, we refer to Gudendorf and Segers [2010].

\section{Logistic model}

The symmetric logistic model in dimension $N$ with parameter $\theta \in(0,1)$ corresponds to the max-stable random vector with cumulative distribution function

$$
\operatorname{pr}[Z \leq z]=\exp \left(-\left(\sum_{j=1}^{N} z_{j}^{-1 / \theta}\right)^{\theta}\right), \quad z=\left(z_{1}, \ldots, z_{N}\right) \in(0, \infty)^{N} .
$$

Proposition 8. Let $\beta=1 / \theta$. In the logistic model (16), the probability measure $P_{j_{0}}$ for any $j_{0}=1, \ldots, N$ is equal to the distribution of the random vector

$$
\left(\frac{F_{1}}{F_{j_{0}}}, \ldots, \frac{F_{N}}{F_{j_{0}}}\right)
$$

where $F_{1}, \ldots, F_{N}$ are independent, $F_{j}, j \neq j_{0}$, follows a Frechet $\left(\beta, c_{\beta}\right)$ distribution with scale parameter $c_{\beta}=\Gamma(1-1 / \beta)^{-1}$ and $\left(F_{j_{0}} / c_{\beta}\right)^{-\beta}$ follows a Gamma $(1-1 / \beta, 1)$ distribution.

REMARK 5. The asymmetric logistic distribution can be represented as the mixture of symmetric logistic distributions; see Theorem 1 in Stephenson [2003], for instance. As a consequence, Proposition 8 also enables exact simulation of asymmetric logistic distributions. 


\section{Negative logistic model}

The negative logistic model in dimension $N$ with parameter $\theta>0$ corresponds to the max-stable random vector $Z$ with cumulative distribution function

$$
\operatorname{pr}[Z \leq z]=\exp \left(\sum_{\emptyset \neq J \subset\{1, \ldots, N\}}(-1)^{|J|}\left(\sum_{j \in J} z_{j}^{\theta}\right)^{-1 / \theta}\right), \quad z \in(0, \infty)^{N} .
$$

Proposition 9. In the negative logistic model (17), the probability measure $P_{j_{0}}$ for any $j_{0}=$ $1, \ldots, N$ is equal to the distribution of the random vector

$$
\left(\frac{W_{1}}{W_{j_{0}}}, \ldots, \frac{W_{N}}{W_{j_{0}}}\right)
$$

where $W_{1}, \ldots, W_{N}$ are independent, $W_{j}, j \neq j_{0}$, follows a Weibull $\left(\theta, c_{\theta}\right)$ distribution with scale parameter $c_{\theta}=\Gamma(1+1 / \theta)^{-1}$ and $\left(W_{j_{0}} / c_{\theta}\right)^{\theta}$ follows a $\Gamma(1+1 / \theta, 1)$ distribution.

\section{Dirichlet mixture model}

The Dirichlet mixture model was introduced by Boldi and Davison [2007]. In dimension $N$, the model corresponds to the max-stable random vector given by

$$
Z=\max _{i \geq 1} \zeta_{i}\left(N Y_{i}\right)
$$

where the $Y_{i}$ 's are independent identically distributed random vectors on the simplex

$$
S_{N-1}=\left\{y \in[0,1]^{n}: \sum_{j=1}^{N} y_{j}=1\right\}
$$

The distribution of each $Y_{i}$ is a mixture of $m$ Dirichlet models, i.e. its Lebesgue density is of the form

$$
h(y)=\sum_{k=1}^{m} \pi_{k} \operatorname{diri}\left(y \mid \alpha_{1 k}, \ldots, \alpha_{N k}\right), \quad y=\left(w_{1}, \ldots, w_{N}\right) \in S_{N-1},
$$

where $\pi_{k} \geq 0, k=1, \ldots, m$ such that $\sum_{k=1}^{m} \pi_{k}=1, \alpha_{i k}>0, i=1, \ldots, N, k=1, \ldots, m$, and

$$
\operatorname{diri}\left(y \mid \alpha_{1}, \ldots, \alpha_{N}\right)=\frac{1}{B(\alpha)} \prod_{j=1}^{N} y_{j}^{\alpha_{j}-1}, \quad B(\alpha)=\frac{\prod_{j=1}^{N} \Gamma\left(\alpha_{j}\right)}{\Gamma\left(\sum_{j=1}^{N} \alpha_{j}\right)} .
$$

Here, the parameters $\pi_{k}$ and $\alpha_{i k}, i=1, \ldots, N, k=1, \ldots, m$, are such that

$$
\mathrm{E}\left[Y_{j}\right]=\sum_{k=1}^{m} \pi_{k} \frac{\alpha_{j k}}{\sum_{i=1}^{N} \alpha_{i k}}=\frac{1}{N}, \quad j=1, \ldots, N .
$$

Proposition 10. In the Dirichlet model (18), we have for any $j_{0}=1, \ldots, N$ that $P_{j_{0}}=$ $\sum_{k=1}^{m} \hat{\pi}_{k} P_{j_{0}}^{(k)}$ where $\hat{\pi}_{k}=\pi_{k} \alpha_{j_{0} k} /\left(\sum_{i=1}^{N} \alpha_{i k}\right)$ and $P_{j_{0}}^{(k)}$ is equal to the distribution of the random vector

$$
\left(\frac{G_{1}^{(k)}}{G_{j_{0}}^{(k)}}, \ldots, \frac{G_{N}^{(k)}}{G_{j_{0}}^{(k)}}\right)
$$

and $G_{1}^{(k)}, \ldots, G_{N}^{(k)}$ are independent random variables with Gamma distribution

$$
G_{j_{0}}^{(k)} \sim \operatorname{Gamma}\left(\alpha_{j_{0} k}+1,1\right) \quad \text { and } G_{j} \sim \operatorname{Gamma}\left(\alpha_{j k}, 1\right), \quad j \neq j_{0} .
$$




\section{Simulation on dense grids}

In many applications, one is interested in simulating a max-stable process $Z$ on a dense grid, e.g. $x=\mathcal{X} \cap(\varepsilon \mathbb{Z})^{d}$. As discussed in Section 4, on average, this requires the simulation of $\mathrm{EC}_{2}(N)=N$ random functions in Algorithm 2, that is, the simulation of $N$ random vectors of size $N$. For small $\varepsilon, N$ will be large and the procedure can become very time consuming. Thus, one might be interested in aborting Algorithm 2 after $m<N$ steps, ensuring exactness of the simulation only at locations $x_{1}, \ldots, x_{m}$. In this case, an alternative design of the algorithm which efficiently chooses the subset of $m$ locations might improve the probability of an exact sample at all $N$ locations.

For comparison of two designs, we introduce the random number

$$
N_{0}=\min \left\{m \in\{1, \ldots, N\}: Z_{m}(x)=Z_{N}(x)\right\} .
$$

For $n \geq N_{0}$, the algorithm does not provide any new extremal functions, but all the simulated functions are rejected. Hence, $N_{0}$ is the optimal number of iterations before aborting the algorithm. One design is preferable to another if its corresponding random number $N_{0}$ tends to be smaller. An efficient design should thus simulate the extremal functions at an early stage of the algorithm. Based on the intuition that $\phi_{x_{n+1}}^{+}$is likely not to be contained in $\Phi_{n}^{+}$if $Z_{n}\left(x_{n+1}\right)$ is small, we propose the following adaptive numbering $x^{(1)}, \ldots, x^{(N)}$ of points in Algorithm 2 ;

$$
\text { set } x^{(1)}=x_{1} \text { and } x^{(n+1)}=\operatorname{argmin}\left\{Z_{n}(x): x \in\left\{x_{1}, \ldots, x_{N}\right\} \backslash\left\{x^{(1)}, \ldots, x^{(n)}\right\}\right\},
$$

for $n=1, \ldots, N-1$. A simulation study indicates that this adaptive version is clearly preferable to Algorithm 2 with a deterministic numbering of locations. The advantage is particularly big in the case of strong dependence which corresponds to simulation on dense grids. More details on the simulation study and its results are provided in the supplementary material to this paper.

\section{Acknowledgement}

Financial support from the Swiss National Science Foundation (second author) and the ANR project McSim (third author) is gratefully acknowledged.

\section{References}

J. Blanchet and A. C. Davison. Spatial modeling of extreme snow depth. Ann. Appl. Stat., 5(3): 1699-1725, 2011.

M.-O. Boldi. A note on the representation of parametric models for multivariate extremes. Extremes, 12:211-218, 2009.

M.-O. Boldi and A. C. Davison. A mixture model for multivariate extremes. J. R. Stat. Soc. Ser. B Stat. Methodol., 69(2):217-229, 2007.

T. A. Buishand, L. de Haan, and C. Zhou. On spatial extremes: with application to a rainfall problem. Ann. Appl. Stat., 2(2):624-642, 2008. 
P. Capéraà, A.-L. Fougères, and C. Genest. Bivariate distributions with given extreme value attractor. J. Multivariate Anal., 72:30-49, 2000.

S. G. Coles. Regional modelling of extreme storms via max-stable processes. J. R. Stat. Soc. Ser. B Stat. Methodol., 55(4):797-816, 1993.

A. C. Davison, S. A. Padoan, and M. Ribatet. Statistical modeling of spatial extremes. Statist. Sci., 27:161-186, 2012.

L. de Haan. A spectral representation for max-stable processes. Ann. Probab., 12(4):1194-1204, 1984.

A. B. Dieker and T. Mikosch. Exact simulation of Brown-Resnick random fields at a finite number of locations. Extremes, pages 1-14, 2015.

C. Dombry and F. Éyi-Minko. Strong mixing properties of max-infinitely divisible random fields. Stochastic Process. Appl., 122(11):3790-3811, 2012.

C. Dombry and F. Éyi-Minko. Regular conditional distributions of continuous max-infinitely divisible random fields. Electron. J. Probab., 18(7):1-21, 2013.

C. Dombry, F. Éyi-Minko, and M. Ribatet. Conditional simulation of max-stable processes. Biometrika, 100(1):111-124, 2013.

S. Engelke, Z. Kabluchko, and M. Schlather. An equivalent representation of the BrownResnick process. Statist. Probab. Lett., 81(8):1150-1154, 2011.

S. Engelke, A. Malinowski, M. Oesting, and M. Schlather. Statistical inference for max-stable processes by conditioning on extreme events. Adv. Appl. Probab., 46:478-495, 2014.

S. Engelke, A. Malinowski, Z. Kabluchko, and M. Schlather. Estimation of Hüsler-Reiss distributions and Brown-Resnick processes. J. R. Stat. Soc. Ser. B Stat. Methodol., 77:239-265, 2015.

K. Ghoudi, A. Khoudraji, and L.-P. Rivest. Propriétés statistiques des copules de valeurs extrêmes bidimensionnelles. Canad. J. Statist., 26:187-197, 1998.

G. Gudendorf and J. Segers. Extreme-value copulas. In Copula theory and its applications, pages 127-145. Springer, 2010.

J. Hüsler and R.-D. Reiss. Maxima of normal random vectors: between independence and complete dependence. Statist. Probab. Lett., 7:283-286, 1989.

Z. Kabluchko. Extremes of independent Gaussian processes. Extremes, 14:285-310, 2011.

Z. Kabluchko, M. Schlather, and L. de Haan. Stationary max-stable fields associated to negative definite functions. Ann. Probab., 37(5):2042-2065, 2009.

M. Oesting and M. Schlather. Conditional sampling for max-stable processes with a mixed moving maxima representation. Extremes, 17(1):157-192, 2014. 
M. Oesting, Z. Kabluchko, and M. Schlather. Simulation of Brown-Resnick processes. Extremes, 15:89-107, 2012.

M. Oesting, M. Schlather, and C. Zhou. On the normalized spectral representation of max-stable processes on a compact set. Available from http://arxiv.org/abs/1310.1813, 2013.

T. Opitz. Extremal $t$ processes: Elliptical domain of attraction and a spectral representation. $J$. Multivar. Anal., 122:409-413, 2013.

S. I. Resnick. Extreme Values, Regular Variation and Point Processes. Springer, New York, 2008.

M. Schlather. Models for stationary max-stable random fields. Extremes, 5(1):33-44, 2002.

R. L. Smith. Max-stable processes and spatial extremes. Unpublished manuscript, 1990.

A. Stephenson. Simulating multivariate extreme value distributions of logistic type. Extremes, 6:49-59, 2003.

E. Thibaud and T. Opitz. Efficient inference and simulation for elliptical Pareto processes. Available from http: / / arxiv.org/abs/1401.0168, 2014. 


\section{A Supplementary material}

\section{A.1 Proof of Proposition 4}

Proof of Proposition 4 In order to analyze the complexity of Algorithm 2, we consider each step of the algorithm separately. In the $n$th step, i.e. for sampling the process perfectly at site $x_{n}$, we simulate Poisson points $\zeta$ and stochastic processes $Y$, until one of the following two conditions is satisfied:

(a) $\zeta<Z_{n-1}\left(x_{n}\right)$. This condition is checked directly after the simulation of $\zeta$ and, in this case, no stochastic process $Y$ needs to be simulated.

(b) $\zeta>Z_{n-1}\left(x_{n}\right)$ and $\zeta Y\left(x_{i}\right) \leq Z\left(x_{i}\right)$ for all $1 \leq i<n-1$. In this case, $Z$ is updated and $\zeta Y$ is an extremal function as it contributes to $Z$ at site $x_{n}$ (and possibly also at some of the sites $\left.x_{n+1}, \ldots, x_{N}\right)$.

Thus, any stochastic process that is simulated is either rejected, i.e. it is not considered as contribution to $Z$ as it does not respect all the values $Z\left(x_{1}\right), \ldots, Z\left(x_{n-1}\right)$, or it leads to an extremal function. Denoting by $\left\{\left(\xi_{i}^{(n)}, \psi_{i}^{(n)}\right), i \geq 1\right\}$ a Poisson point process on $(0, \infty) \times \mathcal{C}$ with intensity measure $\xi^{-2} \mathrm{~d} \xi P_{x_{n}}(\mathrm{~d} \psi)$, the random number $C_{2}(N)$ of processes simulated in Algorithm 2 satisfies

$$
C_{2}(N)=\left|\Phi_{\left\{x_{1}, \ldots, x_{N}\right\}}^{+}\right|+\sum_{n=2}^{N}\left|\left\{i \geq 1: \xi_{i}^{(n)}>Z\left(x_{n}\right), \xi_{i}^{(n)}>\min _{j=1}^{n-1} \frac{Z\left(x_{j}\right)}{\psi_{i}^{(n)}\left(x_{j}\right)}\right\}\right| .
$$

In this formula, the term $\left|\Phi_{\left\{x_{1}, \ldots, x_{N}\right\}}^{+}\right|$is the number of extremal functions that need to be simulated, and the term with index $n$ in the sum is the number of functions that are simulated but rejected since $\xi_{i}^{(n)} \psi_{i}^{(n)}\left(x_{j}\right)>Z\left(x_{j}\right)$ for some $j \leq n-1$. For the computation of the expectation of the second term, conditionally on $\Phi_{\left\{x_{1}, \ldots, x_{n-1}\right\}}^{+}$, i.e. for fixed $Z\left(x_{j}\right), 1 \leq j \leq n-1$, the two sets

$$
\begin{aligned}
\Phi_{1}^{(n)} & =\left\{\left(\xi_{i}^{(n)}, \psi_{i}^{(n)}\right): \xi_{i}^{(n)} \psi_{i}^{(n)}\left(x_{j}\right)>Z\left(x_{j}\right) \text { for some } j=1, \ldots, n-1\right\} \\
\text { and } \Phi_{2}^{(n)} & =\left\{\left(\xi_{i}^{(n)}, \psi_{i}^{(n)}\right): \xi_{i}^{(n)} \psi_{i}^{(n)}\left(x_{j}\right) \leq Z\left(x_{j}\right) \text { for all } j=1, \ldots, n-1\right\}
\end{aligned}
$$

are independent Poisson point processes with intensities $\xi^{-2} 1_{\left\{\xi>\min _{j=1}^{n-1}\left(Z\left(x_{j}\right) / \psi\left(x_{j}\right)\right)\right\}} \mathrm{d} \xi P_{x_{n}}(\mathrm{~d} \psi)$ and $\xi^{-2} \mathbf{1}_{\left\{\xi<\min _{j=1}^{n-1}\left(Z\left(x_{j}\right) / \psi\left(x_{j}\right)\right)\right\}} \mathrm{d} \xi P_{x_{n}}(\mathrm{~d} \psi)$, respectively. Conditioning further on $\Phi_{2}^{(n)}, Z\left(x_{n}\right)$ is also fixed and we obtain

$$
\begin{aligned}
& \mathrm{E}\left(\left|\left\{\left(\xi_{i}^{(n)}, \psi_{i}^{(n)}\right): \xi_{i}^{(n)}>Z\left(x_{n}\right), \xi_{i}^{(n)}>\min _{j=1}^{n-1} \frac{Z\left(x_{j}\right)}{\psi_{i}^{(n)}\left(x_{j}\right)}\right\}\right|\right) \\
= & \mathrm{E}\left(\mathrm{E}\left(\left|\left\{(\xi, \psi) \in \Phi_{1}^{(n)}: \xi>Z\left(x_{n}\right)\right\}\right| \mid \Phi_{\left\{x_{1}, \ldots, x_{n-1}\right\}}^{+}, \Phi_{2}^{(n)}\right)\right) \\
= & \mathrm{E}\left(\iint \xi^{-2} \mathbf{1}_{\left\{\xi>Z\left(x_{n}\right)\right\}} \mathbf{1}_{\left\{\xi>\min _{j=1}^{n-1} \frac{Z\left(x_{j}\right)}{\psi\left(x_{j}\right)}\right\}} \mathrm{d} \xi P_{x_{n}}(\mathrm{~d} \psi)\right)
\end{aligned}
$$




$$
=\mathrm{E}\left(\min \left\{\frac{1}{Z\left(x_{n}\right)}, \max _{j=1}^{n-1} \frac{Y_{n}\left(x_{j}\right)}{Z\left(x_{j}\right)}\right\}\right)
$$

where $Y_{n} \sim P_{x_{n}}$ and $Z$ are independent. The relation $\min \{a, b\}=a+b-\max \{a, b\}, a, b \in \mathbb{R}$, and the fact that $Y_{n}\left(x_{n}\right)=1$ almost surely yield

$$
\begin{aligned}
& \mathrm{E}\left(\left|\left\{\left(\xi_{i}^{(n)}, \psi_{i}^{(n)}\right): \xi_{i}^{(n)}>Z\left(x_{n}\right), \xi_{i}^{(n)}>\min _{j=1}^{n-1} \frac{Z\left(x_{j}\right)}{\psi_{i}^{(n)}\left(x_{j}\right)}\right\}\right|\right) \\
= & \mathrm{E}\left(\frac{1}{Z\left(x_{n}\right)}\right)+\mathrm{E}\left(\max _{j=1}^{n-1} \frac{Y_{n}\left(x_{j}\right)}{Z\left(x_{j}\right)}\right)-\mathrm{E}\left(\max _{j=1}^{n} \frac{Y_{n}\left(x_{j}\right)}{Z\left(x_{j}\right)}\right) \\
= & 1+\mathrm{E}\left|\Phi_{\left\{x_{1}, \ldots, x_{n-1}\right\}}^{+}\right|-\mathrm{E}\left|\Phi_{\left\{x_{1}, \ldots, x_{n}\right\}}^{+}\right|,
\end{aligned}
$$

as $\mathrm{E}\left|\Phi_{\left\{x_{1}, \ldots, x_{n}\right\}}^{+}\right|=\mathrm{E}\left(\max _{j=1}^{n} Y_{n}\left(x_{j}\right) / Z\left(x_{j}\right)\right)$ by Lemma 4.7 in Oesting et al. [2013]. Thus, by (22), we obtain

$$
\begin{aligned}
\mathrm{E} C_{2}(N) & =\mathrm{E}\left|\Phi_{\left\{x_{1}, \ldots, x_{N}\right\}}^{+}\right|+\sum_{n=2}^{N}\left(1+\mathrm{E}\left|\Phi_{\left\{x_{1}, \ldots, x_{n-1}\right\}}^{+}\right|-\mathrm{E}\left|\Phi_{\left\{x_{1}, \ldots, x_{n}\right\}}^{+}\right|\right) \\
& =N-1+\mathrm{E}\left|\Phi_{\left\{x_{1}\right\}}^{+}\right|=N .
\end{aligned}
$$

Moreover, by (2), we have that $\mathrm{E} Z\left(x_{i}\right)^{-1}=1$ for $i=1, \ldots, N$, and, thus,

$$
\mathrm{E}\left(\max _{i=1}^{N} Z\left(x_{i}\right)^{-1}\right) \geq 1
$$

with equality if only if $Z\left(x_{1}\right)=\ldots=Z\left(x_{N}\right)$ holds almost surely.

\section{A.2 Proofs for Section 5}

\section{A.2.1 Moving maximum process}

Proof of Proposition 5 In the case of the moving maximum process (13), the measure $\nu$ associated with the representation (1) is

$$
\nu(A)=\int_{\mathcal{X}} 1_{\{h(\cdot-\chi) \in A\}} \lambda(\mathrm{d} \chi), \quad A \subset \mathcal{C} \text { Borel. }
$$

We deduce from Proposition 1 ,

$$
\begin{aligned}
P_{x_{0}}(A) & =\int_{\mathcal{C}} 1_{\left\{f / f\left(x_{0}\right) \in A\right\}} f\left(x_{0}\right) \nu(\mathrm{d} f)=\int_{\mathcal{X}} 1_{\left\{h(\cdot-\chi) / h\left(x_{0}-\chi\right) \in A\right\}} h\left(x_{0}-\chi\right) \lambda(\mathrm{d} \chi) \\
& =\int_{\mathcal{X}} 1_{\left\{h\left(\cdot+u-x_{0}\right) / h(u) \in A\right\}} h(u) \lambda(\mathrm{d} u)
\end{aligned}
$$

where the last line follows from the simple change of variable $x_{0}-\chi=u$. This proves the result since $h(u) \lambda(\mathrm{d} u)$ is a density function on $\mathcal{X}$. 


\section{A.2.2 Brown-Resnick process}

Our proof of Proposition 6 relies on the following lemma on exponential changes of measures for Gaussian processes.

Lemma 1. The distribution of the random process $(W(x))_{x \in \mathcal{X}}$ under the transformed probability measure $\widehat{\mathrm{pr}}=e^{W\left(x_{0}\right)-\sigma^{2}\left(x_{0}\right) / 2} \mathrm{dpr}$ is equal to the distribution of the Gaussian random process

$$
W(x)+c\left(x_{0}, x\right), \quad x \in \mathcal{X},
$$

where $c(x, y)$ denotes the covariance between $W(x)$ and $W(y)$.

Proof of Lemma 1 . We need to consider finite dimensional distributions only and we compute for some $x_{1}, \ldots, x_{k} \in \mathcal{X}$ the Laplace transform of $\left(W\left(x_{i}\right)\right)_{1 \leq i \leq k}$ under the transformed proba-

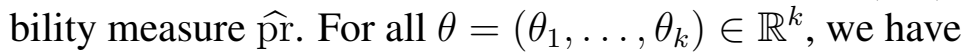

$$
\begin{aligned}
\mathcal{L}\left(\theta_{1}, \ldots, \theta_{k}\right) & =\widehat{\mathrm{E}}\left[e^{\sum_{i=1}^{k} \theta_{i} W\left(x_{i}\right)}\right]=\mathrm{E}\left[e^{W\left(x_{0}\right)-\sigma^{2}\left(x_{0}\right) / 2} e^{\sum_{i=1}^{k} \theta_{i} W\left(x_{i}\right)}\right] \\
& =\exp \left(\frac{1}{2} \tilde{\theta}^{\mathrm{T}} \Sigma \tilde{\theta}-\frac{1}{2} \sigma^{2}\left(x_{0}\right)\right)
\end{aligned}
$$

with $\tilde{\theta}=(1, \theta) \in \mathbb{R}^{k+1}$ and $\tilde{\Sigma}=\left(c\left(x_{i}, x_{j}\right)\right)_{0 \leq i, j \leq k}$ the covariance matrix. We introduce the block decomposition

$$
\tilde{\Sigma}=\left(\begin{array}{cc}
\sigma^{2}\left(x_{0}\right) & \Sigma_{0, k} \\
\Sigma_{k, 0} & \Sigma
\end{array}\right)
$$

with $\Sigma=\left(c\left(x_{i}, x_{j}\right)\right)_{1 \leq i, j \leq k}$ and $\Sigma_{k, 0}=\Sigma_{0, k}^{\mathrm{T}}=\left(c\left(x_{0}, x_{i}\right)\right)_{1 \leq i \leq k}$. The quadratic form in Equation (23) can be rewritten as

$$
\frac{1}{2} \tilde{\theta}^{\mathrm{T}} \tilde{\Sigma} \tilde{\theta}-\frac{1}{2} \sigma^{2}\left(x_{0}\right)=\frac{1}{2}\left(\sigma^{2}\left(x_{0}\right)+\theta^{\mathrm{T}} \Sigma \theta+2 \theta^{\mathrm{T}} \Sigma_{k, 0}\right)-\frac{1}{2} \sigma^{2}\left(x_{0}\right)=\theta^{\mathrm{T}} \Sigma_{k, 0}+\frac{1}{2} \theta^{\mathrm{T}} \Sigma \theta .
$$

We recognize the Laplace transform of a Gaussian random vector with mean $\Sigma_{k, 0}$ and covariance matrix $\Sigma$ whence the Lemma follows.

Proof of Proposition 6 Equations (4) and (14) together with Lemma 1 yield, for all Borel set $A \subset \mathcal{C}$,

$$
\begin{aligned}
P_{x_{0}}(A) & =\int_{\mathcal{C}} 1_{\{f / f(x) \in A\}} f(x) \nu(\mathrm{d} f)=\mathrm{E}\left[e^{W\left(x_{0}\right)-\frac{1}{2} \sigma^{2}\left(x_{0}\right)} 1_{\left\{e^{W(\cdot)-\frac{1}{2} \sigma^{2}(\cdot)} / e^{W\left(x_{0}\right)-\frac{1}{2} \sigma^{2}\left(x_{0}\right)} \in A\right\}}\right] \\
& =\widehat{\operatorname{pr}}\left[\exp \left(W(\cdot)-W\left(x_{0}\right)-\frac{1}{2}\left(\sigma^{2}(\cdot)-\sigma^{2}\left(x_{0}\right)\right)\right) \in A\right] \\
& =\operatorname{pr}\left[\exp \left(W(\cdot)+c\left(x_{0}, \cdot\right)-W\left(x_{0}\right)-c\left(x_{0}, x_{0}\right)-\frac{1}{2}\left(\sigma^{2}(\cdot)-\sigma^{2}\left(x_{0}\right)\right)\right) \in A\right] \\
& =\operatorname{pr}\left[\exp \left(W(\cdot)-W\left(x_{0}\right)-\frac{1}{2}\left(\sigma^{2}(\cdot)+\sigma^{2}\left(x_{0}\right)-2 c\left(x_{0}, \cdot\right)\right)\right) \in A\right] .
\end{aligned}
$$

Using the fact that for all $x \in \mathcal{X}$

$$
\sigma^{2}(x)+\sigma^{2}\left(x_{0}\right)-2 c\left(x_{0}, x\right)=\operatorname{Var}\left[W(x)-W\left(x_{0}\right)\right]
$$


we deduce that $P_{x_{0}}$ is equal to the distribution of the log-normal process

$$
\tilde{Y}(x)=\exp \left(W(x)-W\left(x_{0}\right)-\frac{1}{2} \operatorname{Var}\left[W(x)-W\left(x_{0}\right)\right]\right), \quad x \in \mathcal{X} .
$$

This proves Proposition6.

\section{A.2.3 Extremal- $t$ process}

In the sequel, we write shortly $z^{\alpha}=\max (0, z)^{\alpha}$ for all real numbers $z$.

Lemma 2. The distribution of the random process $\left(W(x) / W\left(x_{0}\right)\right)_{x \in \mathcal{X}}$ under the transformed probability measure $\widehat{\mathrm{pr}}=c_{\alpha} W\left(x_{0}\right)^{\alpha} \mathrm{dpr}$ is equal to the distribution of a Student process with $\alpha+1$ degrees of freedom, location parameter $\mu_{k}$ and scale matrix $\widehat{\Sigma}_{k}$ given by

$$
\mu_{k}=\Sigma_{k, 0} \quad \text { and } \quad \widehat{\Sigma}_{k}=\frac{\Sigma_{k}-\Sigma_{k, 0} \Sigma_{0, k}}{\alpha+1}
$$

where $\Sigma_{k}=\left(c\left(x_{i}, x_{j}\right)\right)_{1 \leq i, j \leq k}$ and $\Sigma_{k, 0}=\Sigma_{0, k}^{\mathrm{T}}=\left(c\left(x_{0}, x_{i}\right)\right)_{1 \leq i \leq k}$.

Proof of Lemma 2 We consider finite dimensional distributions only. Let $k \geq 1$ and $x_{1}, \ldots, x_{k} \in$ $\mathcal{X}$. We first assume that the covariance matrix $\widetilde{\Sigma}=\left(c\left(x_{i}, x_{j}\right)\right)_{0 \leq i, j \leq k}$ is non singular so that $\left(W\left(x_{i}\right)\right)_{0 \leq i \leq k}$ has density

$$
\tilde{g}(y)=(2 \pi)^{-(k+1) / 2} \operatorname{det}(\widetilde{\Sigma})^{-1 / 2} \exp \left(-\frac{1}{2} y^{\mathrm{T}} \widetilde{\Sigma}^{-1} y\right) \quad \text { with } y=\left(y_{i}\right)_{0 \leq i \leq k} .
$$

Setting $z=\left(y_{i} / y_{0}\right)_{1 \leq i \leq k}$, we have for all Borel sets $A_{1}, \ldots, A_{k} \subset \mathbb{R}$

$$
\begin{aligned}
\widehat{\operatorname{pr}}\left[\frac{W\left(x_{i}\right)}{W\left(x_{0}\right)} \in A_{i}, i=1, \ldots, k\right] & =\int_{\mathbb{R}^{k+1}} 1_{\left\{y_{i} / y_{0} \in A_{i}, i=1, \ldots, k\right\}} c_{\alpha} y_{0}^{\alpha} \tilde{g}(\mathbf{y}) \mathrm{d} y \\
& =\int_{\mathbb{R}^{k}} 1_{\left\{z_{i} \in A_{i}, i=1, \ldots, k\right\}}\left(\int_{0}^{\infty} c_{\alpha} y_{0}^{\alpha} \tilde{g}\left(y_{0}, y_{0} z\right) y_{0}^{k} \mathrm{~d} y_{0}\right) \mathrm{d} z
\end{aligned}
$$

We deduce that under $\widehat{\mathrm{pr}}$, the random vector $\left(W\left(x_{i}\right) / W\left(x_{0}\right)\right)_{1 \leq i \leq k}$ has density

$$
\begin{aligned}
g(z) & =\int_{0}^{\infty} c_{\alpha} y_{0}^{k+\alpha} \tilde{g}\left(y_{0}, y_{0} z\right) \mathrm{d} y_{0} \\
& =c_{\alpha}(2 \pi)^{-(k+1) / 2} \operatorname{det}(\widetilde{\Sigma})^{-1 / 2} \int_{0}^{\infty} y_{0}^{k+\alpha} \exp \left(-\frac{\widetilde{z}^{\mathrm{T}} \widetilde{\Sigma^{-1}} \tilde{z}}{2} y_{0}^{2}\right) \mathrm{d} y_{0}
\end{aligned}
$$

with $\tilde{z}=(1, z)$. Using the change of variable $u=\frac{\tilde{z}^{\mathrm{T}} \widetilde{\Sigma}^{-1} \tilde{z}}{2} y_{0}^{2}$, we get

$$
\begin{aligned}
\int_{0}^{\infty} y_{0}^{k+\alpha} \exp \left(-\frac{\tilde{z}^{\mathrm{T}} \widetilde{\Sigma}^{-1} \tilde{z}}{2} y_{0}^{2}\right) \mathrm{d} y_{0} & =\frac{1}{2}\left(\frac{\tilde{z}^{\mathrm{T}} \widetilde{\Sigma}^{-1} \tilde{z}}{2}\right)^{-\frac{\alpha+k+1}{2}} \int_{0}^{\infty} u^{(k+\alpha-1) / 2} \exp (-u) \mathrm{d} u \\
& =\frac{1}{2}\left(\frac{\tilde{z}^{\mathrm{T}} \widetilde{\Sigma}^{-1} \tilde{z}}{2}\right)^{-\frac{\alpha+k+1}{2}} \Gamma\left(\frac{k+\alpha+1}{2}\right)
\end{aligned}
$$


and we obtain after simplification

$$
g(z)=\pi^{-k / 2} \frac{\Gamma\left(\frac{k+\alpha+1}{2}\right)}{\Gamma\left(\frac{\alpha+1}{2}\right)} \operatorname{det}(\widetilde{\Sigma})^{-1 / 2}\left(\tilde{z}^{\mathrm{T}} \widetilde{\Sigma}^{-1} \tilde{z}\right)^{-\frac{\alpha+k+1}{2}} .
$$

Introducing the block decomposition $\widetilde{\Sigma}=\left(\begin{array}{cc}1 & \Sigma_{0, k} \\ \Sigma_{k, 0} & \Sigma_{k}\end{array}\right)$, the inverse matrix is

$$
\widetilde{\Sigma}^{-1}=\left(\begin{array}{cc}
1+\Sigma_{0, k}\left(\Sigma_{k}-\Sigma_{k, 0} \Sigma_{0, k}\right)^{-1} \Sigma_{k, 0} & -\Sigma_{0, k}\left(\Sigma_{k}-\Sigma_{k, 0} \Sigma_{0, k}\right)^{-1} \\
-\left(\Sigma_{k}-\Sigma_{k, 0} \Sigma_{0, k}\right)^{-1} \Sigma_{k, 0} & \left(\Sigma_{k}-\Sigma_{k, 0} \Sigma_{0, k}\right)^{-1}
\end{array}\right) .
$$

By the definition of $\mu_{k}$ and $\widehat{\Sigma}_{k}$, we have

$$
\widetilde{\Sigma}^{-1}=\frac{1}{1+\alpha}\left(\begin{array}{cc}
1+\alpha+\mu_{k}^{\mathrm{T}} \widehat{\Sigma}_{k}^{-1} \mu_{k} & -\mu_{k}^{\mathrm{T}} \widehat{\Sigma}_{k}^{-1} \\
-\widehat{\Sigma}_{k}^{-1} \mu_{k} & \widehat{\Sigma}_{k}^{-1}
\end{array}\right)
$$

and

$$
\tilde{z}^{\mathrm{T}} \widetilde{\Sigma}^{-1} \tilde{z}=(1, z)^{\mathrm{T}} \widetilde{\Sigma}^{-1}(1, z)=\left(1+\frac{\left(z-\mu_{k}\right)^{\mathrm{T}} \widehat{\Sigma}_{k}^{-1}\left(z-\mu_{k}\right)}{\alpha+1}\right)
$$

Finally, we obtain after simplification

$$
g(z)=\pi^{-k / 2}(\alpha+1)^{-k / 2} \frac{\Gamma\left(\frac{k+\alpha+1}{2}\right)}{\Gamma\left(\frac{\alpha+1}{2}\right)} \operatorname{det}\left(\widehat{\Sigma}_{k}\right)^{-1 / 2}\left(1+\frac{\left(z-\mu_{k}\right)^{\mathrm{T}} \widehat{\Sigma}_{k}^{-1}\left(z-\mu_{k}\right)}{\alpha+1}\right)^{-\frac{\alpha+k+1}{2}} .
$$

We recognize the $k$-variate Student density with $\alpha+1$ degrees of freedom, location parameter $\mu$ and scale matrix $\hat{\Sigma}_{k}$.

Proof of Proposition 7 Consider the set

$$
A=\left\{f \in \mathcal{C}_{0} ; f\left(x_{1}\right) \in A_{1}, \cdots, f\left(x_{k}\right) \in A_{k}\right\}
$$

Equations (4) and (15) together with Lemma 2 yield,

$$
\begin{aligned}
P_{x_{0}}(A) & =\int_{\mathcal{C}} 1_{\{f / f(x) \in A\}} f(x) \nu(\mathrm{d} f)=\mathrm{E}\left[c_{\alpha} W\left(x_{0}\right)^{\alpha} 1_{\left\{W\left(x_{i}\right)^{\alpha} / W\left(x_{0}\right)^{\alpha} \in A_{i}, i=1, \ldots, k\right\}}\right] \\
& =\widehat{\operatorname{pr}}\left[W\left(x_{i}\right)^{\alpha} / W\left(x_{0}\right)^{\alpha} \in A_{i}, i=1, \ldots, k\right] \\
& =\operatorname{pr}\left[T_{i}^{\alpha} \in A_{i}, i=1, \ldots, k\right]
\end{aligned}
$$

where $T=\left(T_{1}, \ldots, T_{k}\right)$ has a multivariate Student distribution with $\alpha+1$ degrees of freedom, location parameter $\mu_{k}$ and dispersion matrix $\widehat{\Sigma}_{k}$. This proves the result. 


\section{A.2.4 Multivariate extreme value distributions}

\section{Logistic model}

Proof of Proposition 8 It is easily shown that the logistic model admits the representation

$$
Z=\max _{i \geq 1} \zeta_{i} F_{i}
$$

where the $F_{i}$ 's are independent random vectors with independent Frechet $\left(\beta, c_{\beta}\right)$-distributed components. To check this, we compute

$$
\begin{aligned}
& \mathrm{E}\left[\max _{j=1}^{N} \frac{F_{j}}{z_{j}}\right]=\int_{0}^{\infty} \operatorname{pr}\left[\max _{j=1}^{N} \frac{F_{j}}{z_{j}}>u\right] \mathrm{d} u=\int_{0}^{\infty}\left(1-\prod_{i=1}^{N} \operatorname{pr}\left[F_{j}<z_{j} u\right]\right) \mathrm{d} u \\
= & \int_{0}^{\infty}\left(1-\prod_{i=1}^{N} e^{-\left(z_{j} u / c_{\beta}\right)^{-\beta}}\right) \mathrm{d} u=\int_{0}^{\infty}\left(1-e^{-u^{-\beta} \sum_{j=1}^{N}\left(z_{j} / c_{\beta}\right)^{-\beta}}\right) \mathrm{d} u=\left(\sum_{j=1}^{N} z_{j}^{-\beta}\right)^{1 / \beta} .
\end{aligned}
$$

For the computation of the last integral, we recognize the expectation of a Fréchet distribution. Next we use the fact that $P_{j_{0}}$ is the distribution of $F / F_{j_{0}}$ under the transformed density

$$
y_{j_{0}} \prod_{k=1}^{N} \frac{\beta}{c_{\beta}}\left(\frac{y_{k}}{c_{\beta}}\right)^{-1-\beta} e^{-\left(y_{k} / c_{\beta}\right)^{-\beta}} .
$$

We recognize a product measure where the $j$ th margin, $j \neq j_{0}$, has a $\operatorname{Frechet}\left(\beta, c_{\beta}\right)$ distribution. The $j_{0}$ th marginal has density

$$
y_{j_{0}} \frac{\beta}{c_{\beta}}\left(\frac{y_{j_{0}}}{c_{\beta}}\right)^{-1-\beta} e^{-\left(y_{j_{0}} / c_{\beta}\right)^{-\beta}}
$$

and a simple change of variable reveals that this is the density of $c_{\beta} Z^{-1 / \beta}$ with $Z \sim \operatorname{Gamma}(1-$ $1 / \beta, 1)$.

\section{Negative logistic model}

Proof of Proposition 9 Similarly to the logistic model, we have the spectral representation

$$
Z=\max _{i \geq 1} \zeta_{i} W_{i}
$$

where the $W_{i}$ 's are independent random vectors with independent Weibull $\left(\theta, c_{\theta}\right)$-distributed components with scale parameter $c_{\theta}=\frac{1}{\Gamma(1+1 / \theta)}$. To check this, we compute

$$
\begin{aligned}
\mathrm{E}\left[\max _{j=1}^{N} \frac{W_{j}}{z_{j}}\right]=\int_{0}^{\infty} \operatorname{pr}\left[\max _{j=1}^{N} \frac{W_{j}}{z_{j}}>u\right] \mathrm{d} u=\int_{0}^{\infty}\left(1-\prod_{j=1}^{N} \operatorname{pr}\left[W_{j}<z_{j} u\right]\right) \mathrm{d} u \\
\quad=\int_{0}^{\infty}\left(1-\prod_{j=1}^{N}\left(1-e^{-\left(z_{j} u / c_{\theta}\right)^{\theta}}\right)\right) \mathrm{d} u=-\sum_{J}(-1)^{|J|} \int_{0}^{\infty} e^{-u^{\theta} \sum_{j \in J}\left(z_{j} / c_{\theta}\right)^{\theta}} \mathrm{d} u \\
\quad=-\sum_{J}(-1)^{|J|}\left(\sum_{j \in J}\left(z_{j} / c_{\theta}\right)^{\theta}\right)^{-1 / \theta} \Gamma(1+1 / \theta)=-\sum_{J}(-1)^{|J|}\left(\sum_{j \in J} z_{j}^{\theta}\right)^{-1 / \theta} .
\end{aligned}
$$


For the computation of the last integral, we recognize the expectation of a Weibull distribution. As for the logistic model, $P_{j_{0}}$ is the distribution of $W / W_{j_{0}}$ under the transformed density

$$
y_{j_{0}} \prod_{k=1}^{N} \frac{\theta}{c_{\theta}}\left(\frac{y_{k}}{c_{\theta}}\right)^{\theta-1} e^{-\left(y_{k} / c_{\theta}\right)^{\theta}}
$$

We recognize a product measure where the $j$ th margin, $j \neq j_{0}$ has a Weibull $\left(\theta, c_{\theta}\right)$ distribution. The $j_{0}$ th marginal has density

$$
y_{j_{0}} \frac{\theta}{c_{\theta}}\left(\frac{y_{j_{0}}}{c_{\theta}}\right)^{\theta-1} e^{-\left(y_{j_{0}} / c_{\theta}\right)^{\theta}}
$$

and a simple change of variable reveals that this is the density of $c_{\theta} Z^{1 / \theta}$ with $Z \sim \operatorname{Gamma}(1+$ $1 / \theta, 1)$.

\section{Dirichlet mixture model}

Proof of Proposition 10 . By definition, $P_{j_{0}}$ has the form

$$
\begin{aligned}
P_{j_{0}}(A) & =N \sum_{k=1}^{m} \pi_{k} \int_{S_{N-1}} y_{j_{0}} \mathbf{1}_{\left\{y / y_{j_{0}} \in A\right\}} \operatorname{diri}\left(y \mid \alpha_{1 k}, \ldots, \alpha_{N k}\right) \mathrm{d} y \\
& =N \sum_{k=1}^{m} \hat{\pi}_{k} \frac{\int_{S_{N-1}} y_{j_{0}} \mathbf{1}_{\left\{y / y_{j_{0}} \in A\right\}} \operatorname{diri}\left(y \mid \alpha_{1 k}, \ldots, \alpha_{N k}\right) \mathrm{d} y}{\int_{S_{N-1}} y_{j_{0}} \operatorname{diri}\left(y \mid \alpha_{1 k}, \ldots, \alpha_{N k}\right) \mathrm{d} y}, \quad A \subset(0, \infty)^{N} .
\end{aligned}
$$

Thus, $P_{j_{0}}$ is given as the mixture $P_{j_{0}}=\sum_{k=1}^{m} \hat{\pi}_{k} P_{j_{0}}^{(k)}$, where for each $k=1, \ldots, m$, the probability measure $P_{j_{0}}^{(k)}$ is equal to the distribution of the random vector $\tilde{Y}^{(k)} / \tilde{Y}_{j_{0}}^{(k)}$, and $\tilde{Y}^{(k)}$ has a transformed density proportional to $y_{j_{0}} \prod_{j=1}^{N} y_{j}^{\alpha_{j}-1}$. We recognize the Dirichlet distribution with parameters $\tilde{\alpha}_{1 k}, \ldots, \tilde{\alpha}_{N k}$ given by

$$
\tilde{\alpha}_{j_{0} k}=\alpha_{j_{0} k}+1 \quad \text { and } \quad \tilde{\alpha}_{j k}=\alpha_{j k} \quad j \neq j_{0} .
$$

It is well known that Dirichlet distributions can be expressed in terms of Gamma distributions. More precisely, we have the stochastic representation

$$
\tilde{Y}^{(k)}=\left(G_{1}^{(k)} / \sum_{j=1}^{N} G_{j}^{(k)}, \ldots, G_{N}^{(k)} / \sum_{j=1}^{N} G_{j}^{(k)}\right),
$$

where $G_{j}^{(k)}$ are independent $\operatorname{Gamma}\left(\tilde{\alpha}_{j k}, 1\right)$ random variables. The result follows since $P_{j_{0}}^{(k)}$ is the distribution of $\tilde{Y}^{(k)} / \tilde{Y}_{j_{0}}^{(k)}$. 

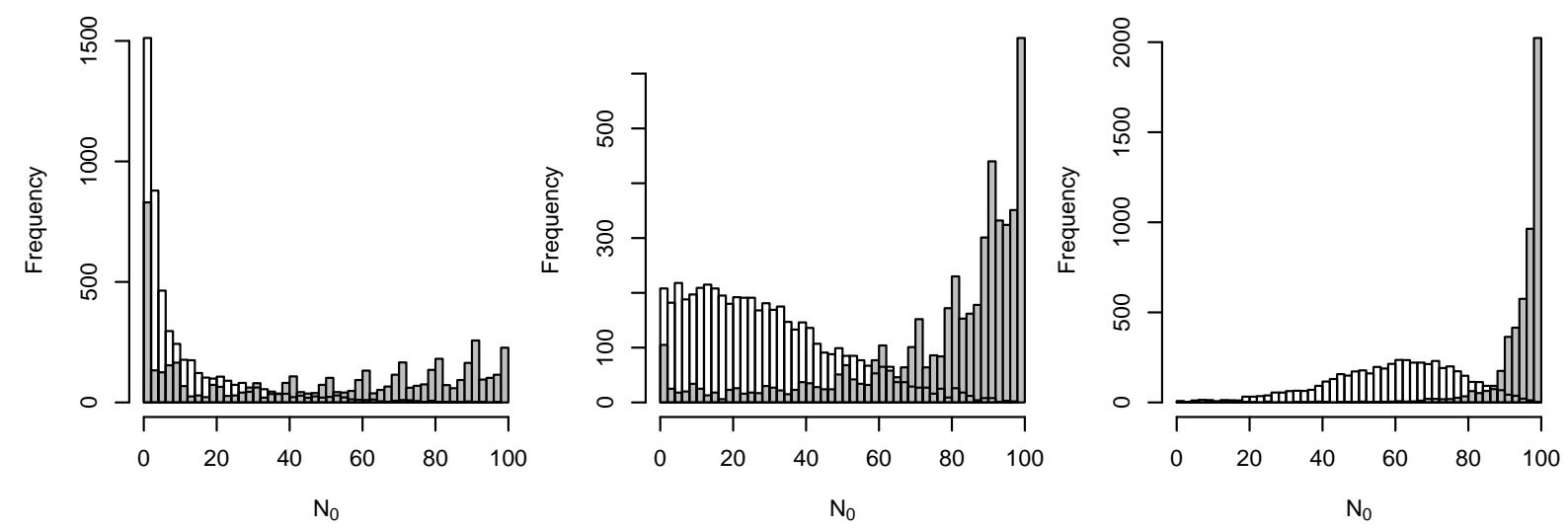

Figure 3: Histogram of $N_{0}$ based on 5000 realizations of a Brown-Resnick process associated to the semi-variogram $\gamma(h)=c\|h\|^{\alpha}$ with $c=1$ and $\alpha=1.5$ (left), $c=2.5$ and $\alpha=1$ (middle) and $c=5$ and $\alpha=0.5$ simulated via Algorithm 2 with the deterministic design (grey) and the adaptive design (21) (white), respectively.

\section{A.3 Simulation Study}

We perform a simulation study to compare the adaptive version of Algorithm 2 introduced in (21) to a version, where the numbering of locations is deterministic. The simulation study is based on 5000 simulations of a Brown-Resnick process associated to a semi-variogram of the type $\gamma(h)=c\|h\|^{\alpha}$ on the two-dimensional grid $\{0.05,0.15, \ldots, 0.95\} \times\{0.05,0.15, \ldots, 0.95\}$. We run Algorithm 2 with the deterministic design (the grid points are ordered by their coordinates in the lexicographical sense) and with the adaptive design (21). The simulation is repeated for different values of the parameter vector $(c, \alpha)$ representing strong dependence $((c, \alpha)=$ $(1,1.5))$, moderate dependence $((c, \alpha)=(2.5,1))$ and weak dependence $((c, \alpha)=(5,0.5))$. The histograms of $N_{0}$ are shown in Figure A.3. For each of the three parameter vectors, the number $N_{0}$ for the adaptive design is stochastically smaller than the corresponding number for the deterministic design. 